\title{
Paper
}

\section{Generation of Arbitrarily-Oriented Ribbed-Pattern Images Using Autocorrelation Coefficient}

\author{
TORU HiRAOKA* ${ }^{\dagger}$ Member
}

(Received December 16, 2018, revised July 08, 2019)

\begin{abstract}
We propose a non-photorealistic rendering (NPR) method for generating arbitrarily-oriented ribbedpattern (AORP) images from gray-scale photographic images. Ribbed patterns consist of wavy lines in a certain orientation. Ribbed-pattern images are obtained by superimposing ribbed patterns and photographic images. AORP images can arbitrarily change the orientation in which ribbed patterns occur. The proposed method is executed using autocorrelation coefficient. The proposed method can automatically generate ribbed patterns according to the change in the density of photographic images, and can arbitrarily change the orientation in which ribbed patterns occur by changing the value of the parameter. In order to verify the effectiveness of the proposed method, we conduct experiments using Lenna image and other photographic images. In the experiments, we show that the proposed method can generate AORP images.
\end{abstract}

Keywords: Non-photorealistic rendering, Ribbed pattern, Autocorrelation coefficient, Arbitrarily orientation

\section{Introduction}

Many computer graphics researchers are exploring NPR techniques for generating images that do not feature photorealism [1] [2]. In recent years, NPR imitates patterns that exist in nature and social life such as cell membrane shapes, zebra stripes, and bamboo weaves [3] [7]. Further, various NPR expressions are required. Such NPR is utilized in the industrial field, the art filed, the entertainment filed, and the like.

In this paper, we focus on ribbed patterns as the patterns in nature and social life, and develop an NPR method to generate AORP images from gray-scale photographic images. Ribbed patterns consist of wavy lines in a certain orientation. Ribbed-pattern images are obtained by superimposing ribbed patterns and photographic images. The proposed method is executed using autocorrelation coefficient. The proposed method can automatically generate ribbed patterns according to the change in the density of photographic images, can arbitrarily change the orientation of ribbed patterns by changing the values of the parameters, and can change the interval of ribbed patterns as well. We conduct experiments using Lenna image and other photographic images. In the experiments, we visually examine the changes in appearance of AORP images by changing the values of the parameters in the proposed method. As a result of the experiments, we clarify that the proposed method is effective for generating AORP images.

As a conventional method for generating NPR images similar to AORP images, there is a method of generating

\footnotetext{
* Corresponding: hiraoka@sun.ac.jp

$\dagger$ Faculty of Information System, University of Nagasaki

Nagasaki-ken, 851-2195 Japan
}

ripple images [8]. The conventional method [8] can generate ripple patterns with gradients of $0,45,90$, and 135 degrees. Ribbed images give a different impression with different textures from ripple images.

\section{Proposed Method}

The proposed method generates AORP images from grayscale photographic images, and is executed in two processes. The first process computes autocorrelation coefficient, and the second process updates images using autocorrelation coefficient. By repeating the first and second processes, AORP images are generated. A flow chart of the proposed method is shown in Figure 1. The detailed procedure of the proposed method is shown as follows.

Step 0 Let the input pixel values on coordinates $(i, j)$ of a gray-scale photographic image be $f_{i, j}$. The pixel values $f_{i, j}$ have value of 256 gradation from 0 to 255 . Let the pixel values of the image at the $t$-th iteration number be $f_{i, j}^{(t)}$, where $f_{i, j}^{(1)}=f_{i, j}$.

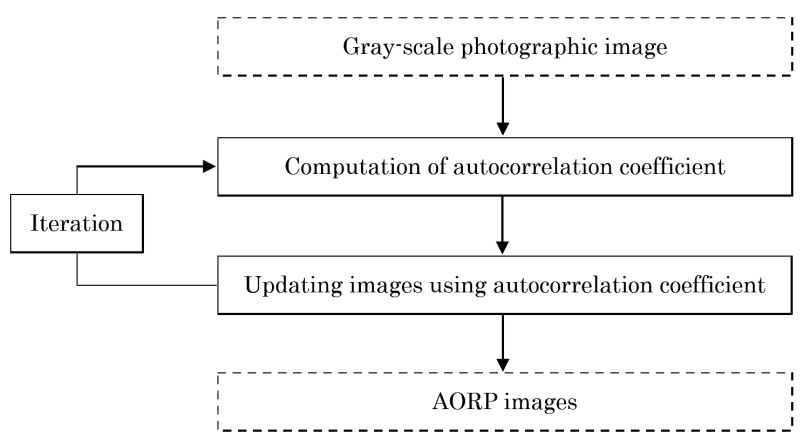

Figure 1: Flow chart of the proposed method. 
Step 1 autocorrelation coefficients $c_{i, j}^{(t)}$ are computed using pixel values within window size $W$ on coordinates $(i, j)$ and $(i+d x, j+d y)$ as the following equations, where $d x$ and $d y$ are integers.

$$
\begin{gathered}
c_{i, j}^{(t)}=\frac{\sum_{k=-W} \sum_{l=-W}^{W} F_{i, j, k, l}^{(t)} F_{i+d x, j+d y, k, l}^{(t)}}{\sqrt{\sum_{k=-W}^{W} \sum_{l=-W}^{W} F_{i, j, k, l}^{(t)}} \sqrt{\sum_{k=-W}^{W} \sum_{l=-W}^{W} F_{i+d x, j+d y, k, l}^{(t)}}} \\
\bar{f}_{i, j}^{(t)}=\frac{\sum_{k=-W} \sum_{l=-W}^{W} f_{i+k, j+l}^{(t)}}{(2 W+1)^{2}} \\
\bar{f}_{i+d x, j+d y}^{(t)}=\frac{\sum_{k=-W}^{W} f_{l=-W}^{W}}{(2 W+1)^{2}} \\
F_{i, j, k, l}^{(t)}=f_{i+k, j+l}^{(t)}-\bar{f}_{i, j}^{(t)} \\
F_{i+d x, j+d y, k, l}^{(t)}=f_{i+d x+k, j+d y+l}^{(t)}-\bar{f}_{i+d x, j+d y}^{(t)}
\end{gathered}
$$

By adjusting $d x$ and $d y$, the orientation of ribbed patterns can be arbitrarily changed. For example, ribbed patterns in the vertical orientation are formed when $d x=1$ and $d y=0$, ribbed patterns in the horizontal orientation are formed when $d x=0$ and $d y=1$, and ribbed patterns in the orientation of 45 degree are formed when $d x=1$ and $d y=1$.

Step 2 the pixel values $f_{i, j}^{(t)}$ are updated to $f_{i, j}^{(t+1)}$ using the autocorrelation coefficients $c_{i, j}^{(t)}$ as the following equation.

$$
f_{i, j}^{(t+1)}= \begin{cases}f_{i, j}^{(t)}-a c_{i, j}^{(t)} & (t \% 2=1) \\ f_{i, j}^{(t)}+a c_{i, j}^{(t)} & (t \% 2=0)\end{cases}
$$

where $a$ is a positive constant and $\%$ is the remainder operator. In case $f_{i, j}^{(t+1)}$ is less than 0 , then $f_{i, j}^{(t+1)}$ must be set to 0 . In case $f_{i, j}^{(t+1)}$ is greater than 255 , then $f_{i, j}^{(t+1)}$ must be set to 255 .

An AORP image is obtained after the above processing of $T$ times iteration.

\section{Experiments}

We conduct two experiments. In the first experiment, we visually confirm AORP images generated by changing the values of the parameters in the proposed method using Lenna image shown in Figure 2. Depending on the usage by the user, the results of the experiment offer guidelines for generating AORP images from various photographic images. In the second experiment, we visually confirm AORP images generated from various photographic images. All photographic images used in the experiments are $512 * 512$ pixels and 256 gradation.
3.1 Experiment with changing parameters AORP images by changing the iteration number $T$ are visually confirmed using Lenna image. The iteration number $T$ is set to 20, 40,60, and 80. Other parameters $a, W, d_{x}$, and $d_{y}$ are set to $32,2,2$, and 2, respectively. The results of the experiment are shown in Fig. 3. As the value of the iteration number $T$ is larger, ribbed patterns become clear and converge.

AORP images by changing the parameter $a$ are confirmed visually. The parameter $a$ is set to $16,32,48$, and 64. Other parameters $T, W, d_{x}$, and $d_{y}$ are set to $80,2,2$, and 2, respectively. The results of the experiment are shown in Figure 4. As the value of the parameter $a$ is larger, ribbed patterns become clear. On the other hand, as the value of the parameter $a$ is larger, it becomes difficult to visually recognize Lenna image.

AORP images by changing the window size $W$ are confirmed visually. The window size $W$ is set to $1,2,3$, and 4 . Other parameters $T, a, d_{x}$, and $d_{y}$ are set to $80,32,2$, and 2 , respectively. The results of the experiment are shown in Figure 5. As the value of the window size $W$ is larger, the

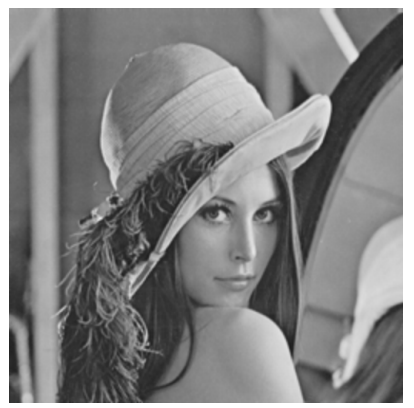

Figure 2: Lenna image.

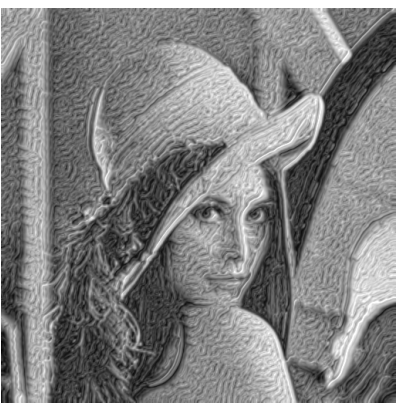

(a) $T=20$

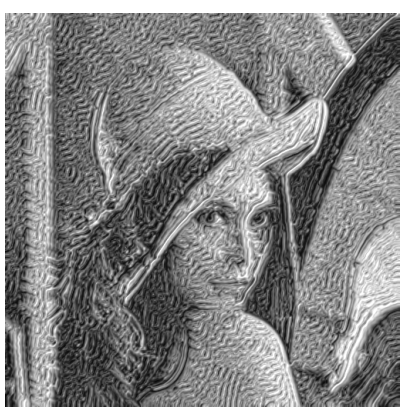

(c) $T=60$

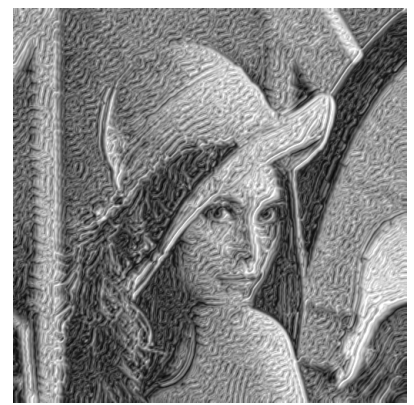

(b) $T=40$

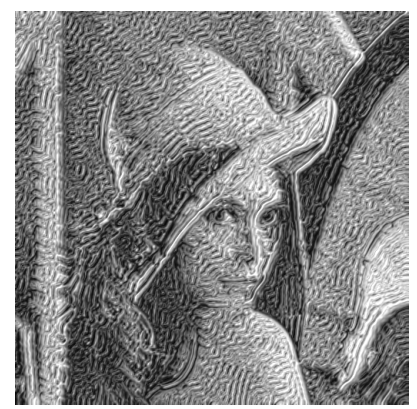

(d) $T=80$
Figure 3: AORP images in the case of the iteration number $T=20,40,60$, and 80 . 


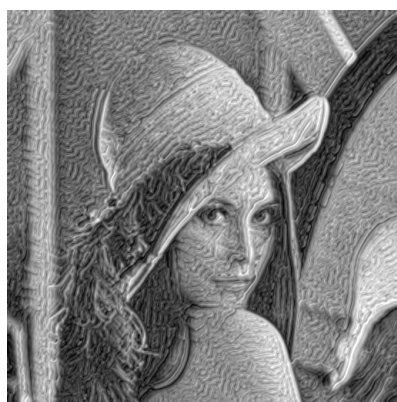

(a) $a=16$

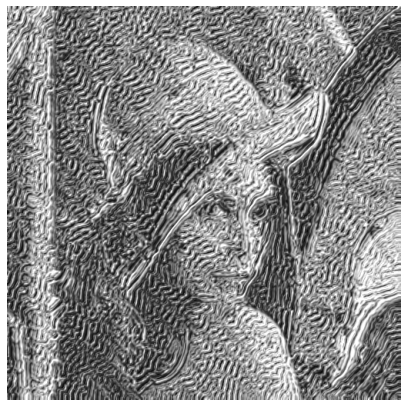

(c) $a=48$

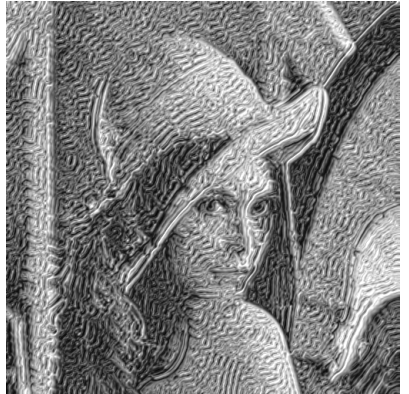

(b) $a=32$

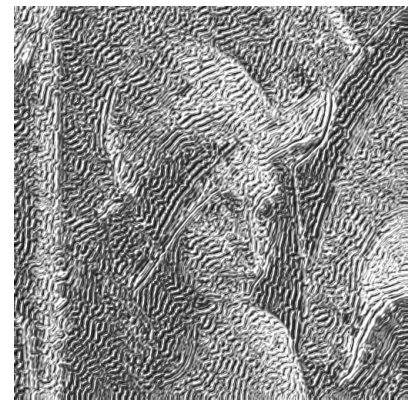

(d) $a=64$

Figure 4: AORP images in the case of the parameter $a=16,32,48$, and 64 .

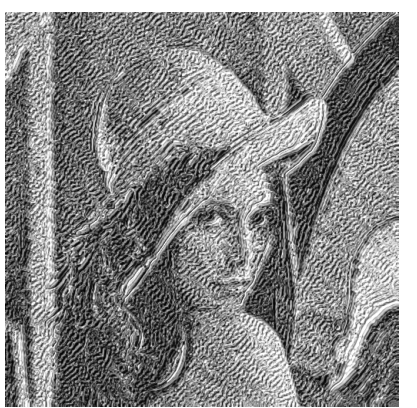

(a) $W=1$

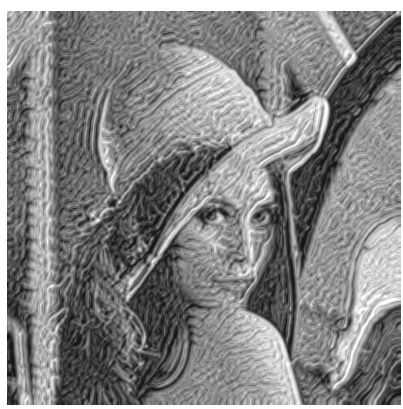

(c) $W=3$

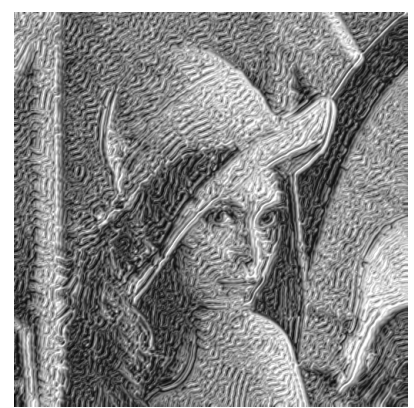

(b) $W=2$

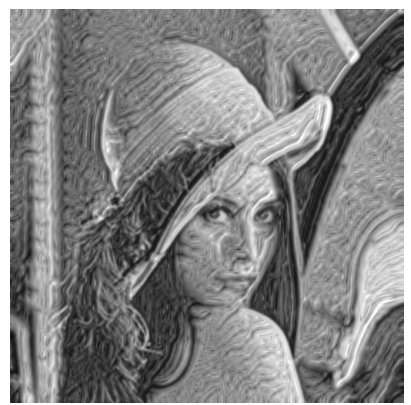

(d) $W=4$

Figure 5: AORP images in the case of the window size $W=1,2,3$, and 4 .

width of ribbed patterns become wider. Then, as the value of the window size $W$ is larger, ribbed patterns are not become clear. In the case of increasing the window size $W$ to make ribbed patterns clear, the value of the parameter $a$ should be increased.

AORP images by changing the parameters $d_{x}$ and $d_{y}$ are confirmed visually. The parameters $d_{x}$ and $d_{y}$ are set to 0

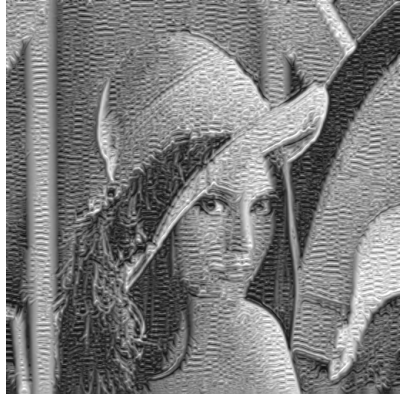

(a) $d_{x}=0, d_{y}=2$

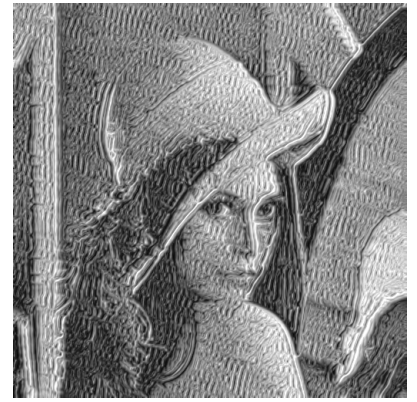

(c) $d_{x}=2, d_{y}=1$

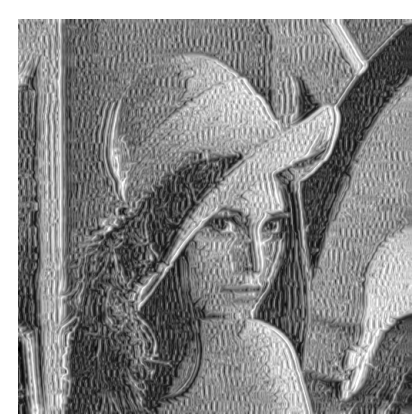

(b) $d_{x}=2, d_{y}=0$

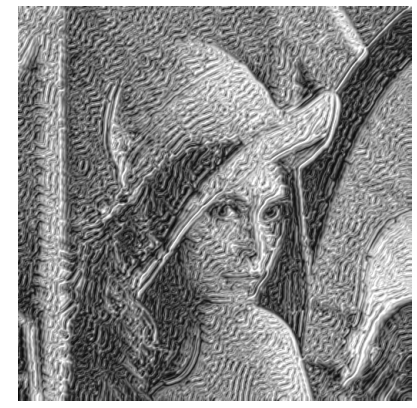

(d) $d_{x}=2, d_{y}=2$
Figure 6: AORP images generated by changing the values of the parameters $d_{x}$ and $d_{y}$.
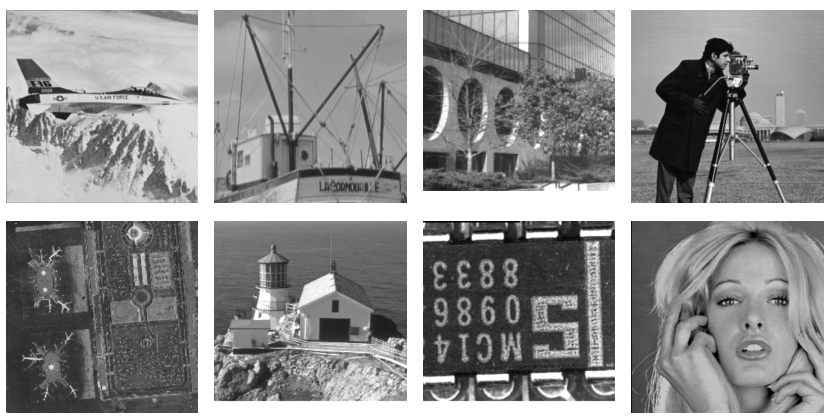

Figure 7: Photographic images.

and 2, 2 and 0,2 and 1, and 2 and 2, respectively. Other parameters $T, a$, and $W$ are set to 80, 32, and 2, respectively. The results of the experiment are shown in Figure 6. By changing the values of the parameters $d_{x}$ and $d_{y}$, it can change the orientation in which ribbed patterns occur arbitrarily. Although the conventional method [8] can not generate ribbed patterns in the direction of $0,45,90$, and 135 degrees, the proposed method can generate ribbed patterns in other directions.

\subsection{Experiment using various photographic images}

The proposed method is applied to eight gray-scale photographic images shown in Figure 7. The parameters $T, a, W$, $d_{x}$, and $d_{y}$ are set to $80,32,2,2$, and 2 , respectively. The results of the experiment are shown in Figure 8. In all AORP images, ribbed patterns can be automatically generated by changes in the density of photographic images.

\section{Conclusion}

We proposed an NPR method for generating AORP images from gray-scale photographic images. The proposed 

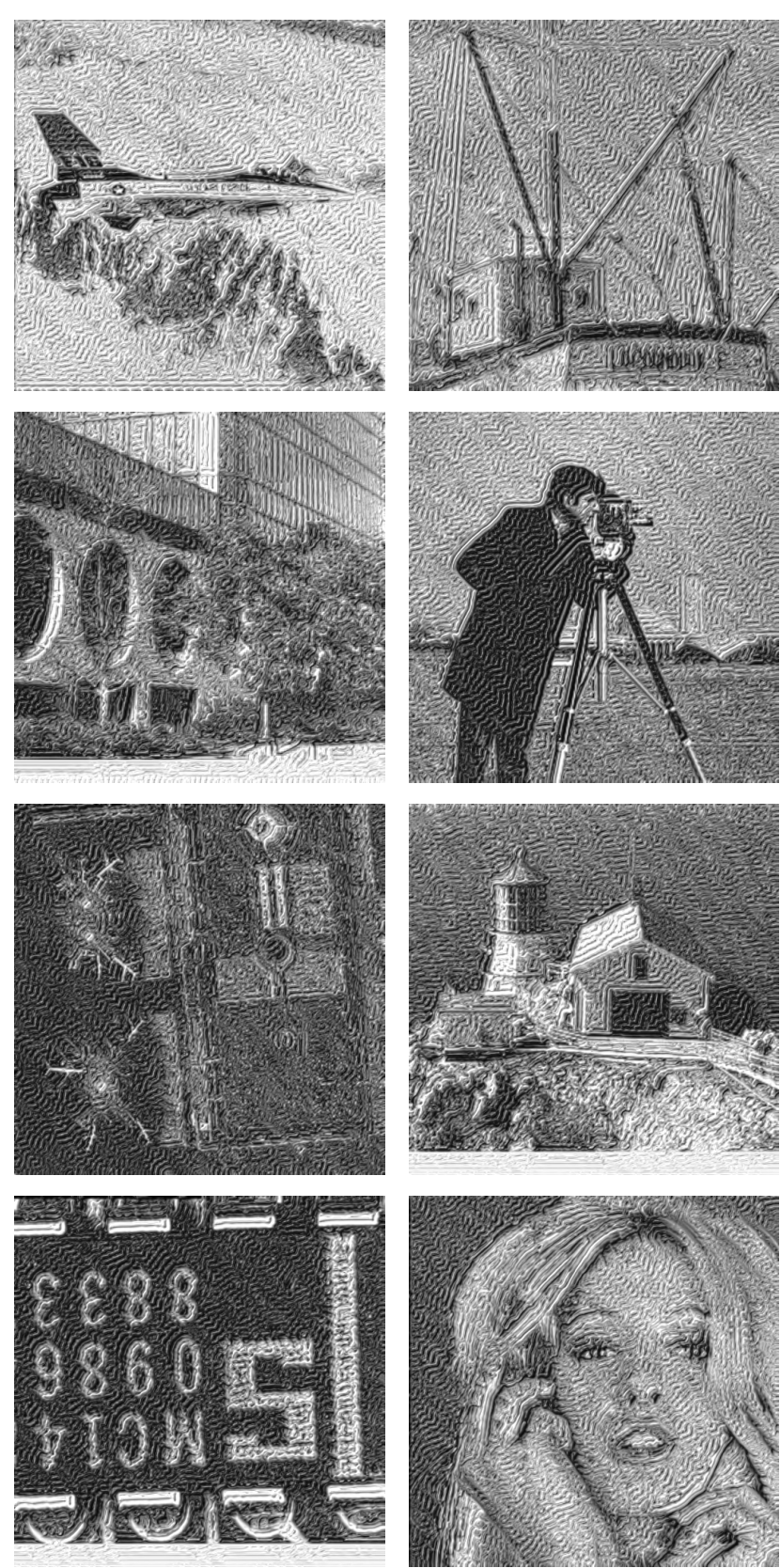

Figure 8: AORP images.

method was executed using autocorrelation coefficient. The proposed method had three features. The first feature is that ribbed patterns can be generated automatically according to the change in the density of photographic images. The second feature is that the orientation of ribbed patterns can be changed arbitrarily by changing the values of the parameters. The third feature is that the interval of ribbed patterns can be changed as well. In the experiments using Lenna image and other photographic images, we clarified that the proposed method can practically realize the three features.

A subject for future study is to expand the proposed method for application to color photographic images and videos.

\section{References}

[1] T. Isenberg, P. Neumann, S. Carpendale, M. Costa Sousa and J. A. Jorge, "Non-Photorealistic Rendering in Context: an Observational Study", IEEE Proceedings of the 4th International Symposium on Non-Photorealistic Animation and Rendering, pp.115-126, 2006.

[2] J. Daniel, S. Erik, Y. Anders and R. Timo, "A survey of Volumetric Illumination Techniques for Interactive Volume Rendering", Computer Graphics Forum, vol.33, no.1, pp.27-51, 2014.

[3] F. Wang, K Inoue and K. Urahama, "Generation of Reversible Bamboo Weave Images", The journal of the Institute of Image Information and Television Engineers, vol.63, no.9, pp.1252-1254, 2009.

[4] A. Werth, "Turing Patterns in Photoshops", Proceedings of Bridges 2015: Mathematics, Music, Art, Architecture, Culture, pp.459-462, 2015.

[5] M. T. Chi, W. C. Liu and S. H. Hsu, "Image Stylization Using Anisotropic Reaction Diffusion", The Visual Computer, vol.32, no.12, pp.1549-1561, 2016.

[6] T. Hiraoka and M. Hirota, "Generation of Cell-Like Color Animation by Inverse Iris Filter", ICIC Express Letters, vol.12, no.1, pp.23-28, 2018.

[7] T. Hiraoka and K. Urahama, "Generation of Bubble Images Using Additive and Multiplication Averages in Different Window Sizes", ICIC Express Letters, vol.13, no.6, pp.469-474, 2019.

[8] T. Hiraoka and K. Urahama, "Generating Ripple-PatternLike Images by Inverse Sobel Filter", The Journal of the Institute of Image Electronics Engineers of Japan, vol.44, no.2, pp.349-352, 2015.

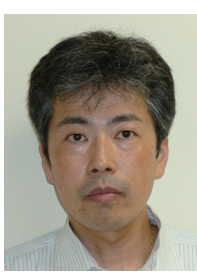

Toru Hiraoka (Member) He received B.Des., M.Des. and D.Eng. degrees from Kyushu Institute of Design in 1995, 1997 and 2005, respectively. He is currently a Professor in University of Nagasaki. His research interests include nonphotorealistic rendering and disaster prevention. 